\title{
CLASSIFICATION OF QUASI-TRIGONOMETRIC SOLUTIONS OF THE CLASSICAL YANG-BAXTER EQUATION
}

\author{
IULIA POP AND ALEXANDER STOLIN
}

\begin{abstract}
It was proved by Montaner and Zelmanov that up to classical twisting Lie bialgebra structures on $\mathfrak{g}[u]$ fall into four classes. Here $\mathfrak{g}$ is a simple complex finite-dimensional Lie algebra. It turns out that classical twists within one of these four classes are in a one-to-one correspondence with the so-called quasi-trigonometric solutions of the classical Yang-Baxter equation. In this paper we give a complete list of the quasi-trigonometric solutions in terms of sub-diagrams of the certain Dynkin diagrams related to $\mathfrak{g}$. We also explain how to quantize the corresponding Lie bialgebra structures.
\end{abstract}

\section{INTRODUCTION}

The present paper constitutes a step towards the classification of quantum groups. We describe an algorithm for the quantization of all Lie bialgebra structures on the polynomial Lie algebra $P=\mathfrak{g}[u]$, where $\mathfrak{g}$ is a simple complex finite-dimensional Lie algebra.

Lie bialgebra structures on $P$, up to so-called classical twisting, have been classified by F. Montaner and E. Zelmanov in [9. We recall that given a Lie co-bracket $\delta$ on $P$, a classical twist is an element $s \in P \wedge P$ such that

$$
\mathrm{CYB}(s)+\operatorname{Alt}(\delta \otimes \mathrm{id})(s)=0,
$$

where CYB is the l.h.s. of the classical Yang-Baxter equation.

We also note that a classical twist does not change the classical double $D_{\delta}(P)$ associated to a given Lie bialgebra structure $\delta$. If $\delta^{s}$ is the twisting co-bracket via $s$, then the Lie bialgebras $(P, \delta)$ and $\left(P, \delta^{s}\right)$ are in the same class, i.e. there exists a Lie algebra isomorphism between $D_{\delta}(P)$ and $D_{\delta^{s}}(P)$, preserving the canonical forms and compatible with the canonical embeddings of $P$ into the doubles.

According to the results of Montaner and Zelmanov, there are four Lie bialgebra structures on $P$ up to classical twisting. Let us present them:

Case 1. Consider $\delta_{1}=0$. Consequently, $D_{1}(P)=P+\varepsilon P^{*}$, where $\varepsilon^{2}=0$. The symmetric nondegenerate invariant form $Q$ is given by the canonical pairing between $P$ and $\varepsilon P^{*}$.

Lie bialgebra structures which fall in this class are the elements $s \in P \wedge P$ satisfying $\mathrm{CYB}(s)=0$. Such elements are in a one-to-one correspondence with finite-dimensional quasi-Frobenius Lie subalgebras of $P$.

Case 2. Let us consider the co-bracket $\delta_{2}$ given by

$$
\delta_{2}(p(u))=\left[r_{2}(u, v), p(u) \otimes 1+1 \otimes p(v)\right],
$$

where $r_{2}(u, v)=\Omega /(u-v)$. Here $\Omega$ denotes the quadratic Casimir element on $\mathfrak{g}$.

It was proved in [10] that the associated classical double is $D_{2}(P)=\mathfrak{g}\left(\left(u^{-1}\right)\right)$, together with the canonical invariant form

$$
Q(f(u), g(u))=\operatorname{Res}_{u=0} K(f, g),
$$

2000 Mathematics Subject Classification. Primary 17B37, 17B62; Secondary 17B81.

Key words and phrases. Classical Yang-Baxter equation, $r$-matrix, Manin triple, parabolic subalgebra, generalized Belavin-Drinfeld data. 
where $K$ denotes the Killing form of the Lie algebra $\mathfrak{g}\left(\left(u^{-1}\right)\right)$ over $\mathbb{C}\left(\left(u^{-1}\right)\right)$.

Moreover, the Lie bialgebra structures which are obtained by twisting $\delta_{2}$ are in a one-to-one correspondence with so-called rational solutions of the CYBE, according to [10].

Case 3. In this case, let us consider the Lie bialgebra structure given by

$$
\delta_{3}(p(u))=\left[r_{3}(u, v), p(u) \otimes 1+1 \otimes p(v)\right],
$$

with $r_{3}(u, v)=v \Omega /(u-v)+\Sigma_{\alpha} e_{\alpha} \otimes f_{\alpha}+\frac{1}{2} \Omega_{0}$, where $e_{\alpha}, f_{\alpha}$ are root vectors of $\mathfrak{g}$ and $\Omega_{0}$ is the Cartan part of $\Omega$.

It was proved in [6] that the associated classical double is $D_{3}(P)=\mathfrak{g}\left(\left(u^{-1}\right)\right) \times \mathfrak{g}$, together with the invariant nondegenerate form $Q$ defined by

$$
Q((f(u), a),(g(u), b))=K(f(u), g(u))_{0}-K(a, b),
$$

where the index zero means that one takes the free term in the series expansion. According to [6, there is a one-to-one correspondence between Lie bialgebra structures which are obtained by twisting $\delta_{3}$ and so-called quasi-trigonometric solutions of the CYBE.

Case 4. We consider the co-bracket on $P$ given by

$$
\delta_{4}(p(u))=\left[r_{4}(u, v), p(u) \otimes 1+1 \otimes p(v)\right],
$$

with $r_{4}(u, v)=u v \Omega /(v-u)$.

It was shown in [13 that the classical double associated to the Lie bialgebra structure $\delta_{4}$ is $D_{4}(P)=$ $\mathfrak{g}\left(\left(u^{-1}\right)\right) \times(\mathfrak{g} \otimes \mathbb{C}[\varepsilon])$, where $\varepsilon^{2}=0$. The form $Q$ is described as follows: if $f(u)=\sum_{-\infty}^{N} a_{k} u^{k}$ and $g(u)=\sum_{-\infty}^{N} b_{k} u^{k}$, then

$$
Q\left(f(u)+A_{0}+A_{1} \varepsilon, g(u)+B_{0}+B_{1} \varepsilon\right)=\operatorname{Res}_{u=0} u^{-2} K(f, g)-K\left(A_{0}, B_{1}\right)-K\left(A_{1}, B_{0}\right) .
$$

Lie bialgebra structures which are in the same class as $\delta_{4}$ are in a one-to-one correspondence with quasi-rational r-matrices, as it was proved in [13].

Regarding the quantization of these Lie bialgebra structures on $P$, the following conjecture stated in [6] and proved by G. Halbout in 4] plays a crucial role.

Theorem 1.1. Any classical twist can be extended to a quantum twist, i.e., if $(L, \delta)$ is any Lie bialgebra, $s$ is a classical twist, and $(A, \Delta, \varepsilon)$ is a quantization of $(L, \delta)$, there exists $F \in A \otimes A$ such that

(1) $F=1+O(\hbar)$ and $F-F^{21}=\hbar s+O\left(\hbar^{2}\right)$,

(2) $(\Delta \otimes \mathrm{id})(F) F^{12}-(\mathrm{id} \otimes \Delta)(F) F^{23}=0$,

(3) $(\varepsilon \otimes$ id $)(F)=($ id $\otimes \varepsilon)(F)=1$.

Moreover gauge equivalence classes of quantum twists for $A$ are in bijection with gauge equivalence classes of $\hbar$-dependent classical twists $s_{\hbar}=\hbar s_{1}+O\left(\hbar^{2}\right)$ for $L$.

Let us suppose that we have a Lie bialgebra structure $\delta$ on $P$. Then $\delta$ is obtained by twisting one of the four structures $\delta_{i}$ from Cases 1-4. This above theorem implies that in order to find a quantization for $(P, \delta)$, it is sufficient to determine the quantization of $\delta_{i}$ and then find the quantum twist whose classical limit is $s$. Let us note that the quantization of $\left(P, \delta_{3}\right)$ is well-known. The corresponding quantum algebra was introduced by $\mathrm{V}$. Tolstoy in [14] and it is denoted by $U_{q}(\mathfrak{g}[u])$.

The quasi-trigonometric solutions of the CYBE were studied in 77, where it was proved that they fall into classes, which are in a one-to-one correspondence with vertices of the extended Dynkin diagram of $\mathfrak{g}$. Let us consider corresponding roots, namely simple roots $\alpha_{1}, \alpha_{2}, \cdots \alpha_{r}$ and $\alpha_{0}=-\alpha_{\max }$. In 7] quasi-trigonometric solutions corresponding to the simple roots which have coefficient one in the decomposition of the maximal root were classified. It was also proved there that quasi-trigonometric solutions corresponding to $\alpha_{0}$ are in a one-to-one correspondence with constant solutions of the modified CYBE classified in [1] and the polynomial part of these solutions is constant. The aim of our paper 
is to obtain a complete classification of quasi-trigonometric solutions of the CYBE. In particular, we describe all the quasi-trigonometric solutions with non-trivial polynomial part for $\mathfrak{g}=o(5)$.

\section{LiE BIALGEBRA STRUCTURES ASSOCIATED WITH QUASI-TRIGONOMETRIC SOLUTIONS}

Definition 2.1. A solution $X$ of the CYBE is called quasi-trigonometric if it is of the form $X(u, v)=$ $v \Omega /(u-v)+p(u, v)$, where $p$ is a polynomial with coefficients in $\mathfrak{g} \otimes \mathfrak{g}$.

The class of quasi-trigonometric solutions is closed under gauge transformations. We first need to introduce the following notation: Let $R$ be a commutative ring and let $L$ be a Lie algebra over $R$. Let us denote by $\operatorname{Aut}_{R}(L)$ the group of automorphisms of $L$ over $R$. In other words we consider such automorphisms of $L$, which satisfy the condition $f(r l)=r f(l)$, where $r \in R, l \in L$.

At this point we note that there exists a natural embedding

$$
\operatorname{Aut}_{\mathbb{C}[u]}(\mathfrak{g}[u]) \hookrightarrow \operatorname{Aut}_{\mathbb{C}\left(\left(u^{-1}\right)\right)}\left(\mathfrak{g}\left(\left(u^{-1}\right)\right)\right),
$$

defined by the formula

$$
\sigma\left(u^{-k} x\right)=u^{-k} \sigma(x)
$$

for any $\sigma \in \operatorname{Aut}_{\mathbb{C}[u]}(\mathfrak{g}[u])$ and $x \in \mathfrak{g}[u]$.

Now if $X$ is a quasi-trigonometric solution and $\sigma(u) \in \operatorname{Aut}_{\mathbb{C}[u]}(\mathfrak{g}[u])$, one can check that the function $Y(u, v):=(\sigma(u) \otimes \sigma(v))(X(u, v))$ is again a quasi-trigonometric solution. $X$ and $Y$ are said to be gauge equivalent.

Theorem 2.2. There exists a natural one-to-one correspondence between quasi-trigonometric solutions of CYBE for $\mathfrak{g}$ and linear subspaces $W$ of $\mathfrak{g}\left(\left(u^{-1}\right)\right) \times \mathfrak{g}$ which satisfy the following properties:

(1) $W$ is a Lie subalgebra of $\mathfrak{g}\left(\left(u^{-1}\right)\right) \times \mathfrak{g}$ and $W \supseteq u^{-N} \mathfrak{g}\left[\left[u^{-1}\right]\right]$ for some positive integer $N$.

(2) $W \oplus \mathfrak{g}[u]=\mathfrak{g}\left(\left(u^{-1}\right)\right) \times \mathfrak{g}$.

(3) $W$ is a Lagrangian subspace of $\mathfrak{g}\left(\left(u^{-1}\right)\right) \times \mathfrak{g}$ with respect to the invariant bilinear form $Q$ given by (1.5).

Let $\sigma(u) \in \operatorname{Aut}_{\mathbb{C}[u]}(\mathfrak{g}[u])$. Let $\widetilde{\sigma}(u)=\sigma(u) \oplus \sigma(0)$ be the induced automorphism of $\mathfrak{g}\left(\left(u^{-1}\right)\right) \times \mathfrak{g}$.

Definition 2.3. We will say that $W_{1}$ and $W_{2}$ are gauge equivalent if there exists $\sigma(u) \in \operatorname{Aut}_{\mathbb{C}[u]}(\mathfrak{g}[u])$ such that $W_{1}=\tilde{\sigma}(u) W_{2}$.

It was checked in [6] that two quasi-trigonometric solutions are gauge equivalent if and only if the corresponding subalgebras are gauge equivalent.

Let $\mathfrak{h}$ be a Cartan subalgebra of $\mathfrak{g}$ with the corresponding set of roots $R$ and a choice of simple roots $\Gamma$. Denote by $\mathfrak{g}_{\alpha}$ the root space corresponding to a root $\alpha$. Let $\mathfrak{h}(\mathbb{R})$ be the set of all $h \in \mathfrak{h}$ such that $\alpha(h) \in \mathbb{R}$ for all $\alpha \in R$. Consider the valuation on $\mathbb{C}\left(\left(u^{-1}\right)\right)$ defined by $v\left(\sum_{k \geq n} a_{k} u^{-k}\right)=n$. For any root $\alpha$ and any $h \in \mathfrak{h}(\mathbb{R})$, set $M_{\alpha}(h):=\left\{f \in \mathbb{C}\left(\left(u^{-1}\right)\right): v(f) \geq \alpha(h)\right\}$. Consider

$$
\mathbb{O}_{h}:=\mathfrak{h}\left[\left[u^{-1}\right]\right] \oplus\left(\oplus_{\alpha \in R} M_{\alpha}(h) \otimes \mathfrak{g}_{\alpha}\right) .
$$

As it was shown in [7, any maximal order $W$ which corresponds to a quasi-trigonometric solution of the CYBE, can be embedded (up to some gauge equivalence) into $\mathbb{O}_{h} \times \mathfrak{g}$. Moreover $h$ may be taken as a vertex of the standard simplex $\Delta_{s t}=\left\{h \in \mathfrak{h}(\mathbb{R}): \alpha(h) \geq 0\right.$ for all $\alpha \in \Gamma$ and $\left.\alpha_{\max } \leq 1\right\}$.

Vertices of the above simplex correspond to vertices of the extended Dynkin diagram of $\mathfrak{g}$, the correspondence being given by the following rule:

$$
\begin{gathered}
0 \leftrightarrow \alpha_{\max } \\
h_{i} \leftrightarrow \alpha_{i},
\end{gathered}
$$

where $\alpha_{i}\left(h_{j}\right)=\delta_{i j} / k_{j}$ and $k_{j}$ are given by the relation $\sum k_{j} \alpha_{j}=\alpha_{\max }$. We will write $\mathbb{O}_{\alpha}$ instead of $\mathbb{O}_{h}$ if $\alpha$ is the root which corresponds to the vertex $h$.

By straightforward computations, one can check the following two results: 
Lemma 2.4. Let $R$ be the set of all roots and $\alpha$ an arbitrary simple root. Let $k$ be the coefficient of $\alpha$ in the decomposition of $\alpha_{\max }$.

For each $r,-k \leq r \leq k$, let $R_{r}$ denote the set of all roots which contain $\alpha$ with coefficient $r$. Let $\mathfrak{g}_{0}=\mathfrak{h} \oplus \sum_{\beta \in R_{0}} \mathfrak{g}_{\beta}$ and $\mathfrak{g}_{r}=\sum_{\beta \in R_{r}} \mathfrak{g}_{\beta}$. Then

$$
\mathbb{O}_{\alpha}=\sum_{r=1}^{k} u^{-1} \mathbb{O} \mathfrak{g}_{r}+\sum_{r=1-k}^{0} \mathbb{O} \mathfrak{g}_{r}+u \mathbb{O} \mathfrak{g}_{-k},
$$

where $\mathbb{O}:=\mathbb{C}\left[\left[u^{-1}\right]\right]$.

Lemma 2.5. Let $\alpha$ be a simple root and $k$ its coefficient in the decomposition of $\alpha_{\max }$. Let $\Delta_{\alpha}$ denote the set of all pairs $(a, b), a \in \mathfrak{g}_{0}+\mathfrak{g}_{-k}, b \in \mathfrak{g}_{0}+\mathfrak{g}_{-1}+\ldots+\mathfrak{g}_{-k}, a=a_{0}+a_{-k}, b=b_{0}+b_{-1}+\ldots+b_{-k}$ and $a_{0}=b_{0}$. Then

(i) The orthogonal complement of $\mathbb{O}_{\alpha} \times \mathfrak{g}$ with respect to $Q$ is given by

$$
\left(\mathbb{O}_{\alpha} \times \mathfrak{g}\right)^{\perp}=\sum_{r=-k}^{-1} \mathbb{O} \mathfrak{g}_{r}+\sum_{r=0}^{k-1} u^{-1} \mathbb{O} \mathfrak{g}_{r}+u^{-2} \mathbb{O} \mathfrak{g}_{k}
$$

(ii) There exists an isomorphism $\sigma$

$$
\frac{\mathbb{O}_{\alpha} \times \mathfrak{g}}{\left(\mathbb{O}_{\alpha} \times \mathfrak{g}\right)^{\perp}} \cong\left(\mathfrak{g}_{k} \oplus \mathfrak{g}_{0} \oplus \mathfrak{g}_{-k}\right) \times \mathfrak{g}
$$

given by

$$
\sigma\left((f, a)+\left(\mathbb{O}_{\alpha} \times \mathfrak{g}\right)^{\perp}\right)=\left(a_{0}+b_{0}+c_{0}, a\right),
$$

where the element $f \in \mathbb{O}_{\alpha}$ is decomposed according to Lemma 2.4:

$$
f=u^{-1}\left(a_{0}+a_{1} u^{-1}+\ldots\right)+\left(b_{0}+b_{1} u^{-1}+\ldots\right)+u\left(c_{0}+c_{1} u^{-1}+\ldots\right)+\ldots,
$$

$a_{i} \in \mathfrak{g}_{k}, b_{i} \in \mathfrak{g}_{0}, c_{i} \in \mathfrak{g}_{-k}$ and $a \in \mathfrak{g}$.

(iii) $\left(\mathbb{O}_{\alpha} \times \mathfrak{g}\right) \cap \mathfrak{g}[u]$ is sent via the isomorphism $\sigma$ to $\Delta_{\alpha}$.

Let us make an important remark. The Lie subalgebra $\mathfrak{g}_{k}+\mathfrak{g}_{0}+\mathfrak{g}_{-k}$ of $\mathfrak{g}$ coincides with the semisimple Lie algebra whose Dynkin diagram is obtained from the extended Dynkin diagram of $\mathfrak{g}$ by crossing out $\alpha$. Let us denote this subalgebra by $L_{\alpha}$. The Lie algebra $L_{\alpha} \times \mathfrak{g}$ is endowed with the following invariant bilinear form:

$$
Q^{\prime}((a, b),(c, d))=K(a, c)-K(b, d),
$$

for any $a, c \in L_{\alpha}$ and $b, d \in \mathfrak{g}$.

On the other hand, $\mathfrak{g}_{0}+\mathfrak{g}_{-k}$ is the parabolic subalgebra $P_{-\alpha_{\max }}^{+}$of $L_{\alpha}$ which corresponds to $-\alpha_{\max }$. The Lie subalgebra $\mathfrak{g}_{0}+\mathfrak{g}_{-1}+\ldots+\mathfrak{g}_{-k}$ is the parabolic subalgebra $P_{\alpha}^{-}$of $\mathfrak{g}$ which corresponds to the root $\alpha$ and contains the negative Borel subalgebra. Let us also note that $\mathfrak{g}_{0}$ is precisely the reductive part of $P_{\alpha}^{-}$and of $P_{-\alpha_{\max }}^{+}$. We can conclude that the set $\Delta_{\alpha}$ consists of all pairs $(a, b) \in P_{-\alpha_{\max }}^{+} \times P_{\alpha}^{-}$ whose reductive parts are equal.

Theorem 2.6. Let $\alpha$ be a simple root. There is a one-to-one correspondence between Lagrangian subalgebras $W$ of $\mathfrak{g}\left(\left(u^{-1}\right)\right) \times \mathfrak{g}$ which are contained in $\mathbb{O}_{\alpha} \times \mathfrak{g}$ and transversal to $\mathfrak{g}[u]$, and Lagrangian subalgebras $\mathfrak{l}$ of $L_{\alpha} \times \mathfrak{g}$ transversal to $\Delta_{\alpha}$ (with respect to the bilinear form $Q^{\prime}$ ).

Proof. Since $W$ is a subspace of $\mathbb{O}_{\alpha} \times \mathfrak{g}$, let $\mathfrak{l}$ be its image in $L_{\alpha} \times \mathfrak{g}$. Because $W$ is transversal to $\mathfrak{g}[u]$, one can check that $\mathfrak{l}$ is transversal to the image of $\left(\mathbb{O}_{\alpha} \times \mathfrak{g}\right) \cap \mathfrak{g}[u]$ in $L_{\alpha} \times \mathfrak{g}$, which is exactly $\Delta_{\alpha}$. The fact that $W$ is Lagrangian implies that $\mathfrak{l}$ is also Lagrangian.

Conversely, if $\mathfrak{l}$ is a Lagrangian subalgebra of $L_{\alpha} \times \mathfrak{g}$ transversal to $\Delta_{\alpha}$, then its preimage $W$ in $\mathbb{O}_{\alpha} \times \mathfrak{g}$ is transversal to $\mathfrak{g}[u]$ and Lagrangian as well. 
The Lagrangian subalgebras $\mathfrak{l}$ of $L_{\alpha} \times \mathfrak{g}$ which are transversal to $\Delta_{\alpha}$, can be determined using results of P. Delorme [2] on the classification of Manin triples. We are interested in determining Manin triples of the form $\left(Q^{\prime}, \Delta_{\alpha}, \mathfrak{l}\right)$.

Let us recall Delorme's construction of so-called generalized Belavin-Drinfeld data. Let $\mathfrak{r}$ be a finitedimensional complex, reductive, Lie algebra and $B$ a symmetric, invariant, nondegenerate bilinear form on $\mathfrak{r}$. The goal in 22 is to classify all Manin triples of $\mathfrak{r}$ up to conjugacy under the action on $\mathfrak{r}$ of the simply connected Lie group $\mathcal{R}$ whose Lie algebra is $\mathfrak{r}$.

One denotes by $\mathfrak{r}_{+}$and $\mathfrak{r}_{-}$respectively the sum of the simple ideals of $\mathfrak{r}$ for which the restriction of $B$ is equal to a positive (negative) multiple of the Killing form. Then the derived ideal of $\mathfrak{r}$ is the sum of $\mathfrak{r}_{+}$and $\mathfrak{r}_{-}$.

Let $\mathfrak{j}_{0}$ be a Cartan subalgebra of $\mathfrak{r}, \mathfrak{b}_{0}$ a Borel subalgebra containing $\mathfrak{j}_{0}$ and $\mathfrak{b}_{0}^{\prime}$ be its opposite. Choose $\mathfrak{b}_{0} \cap \mathfrak{r}_{+}$as Borel subalgebra of $\mathfrak{r}_{+}$and $\mathfrak{b}_{0}^{\prime} \cap \mathfrak{r}_{-}$as Borel subalgebra of $\mathfrak{r}_{-}$. Denote by $\Sigma_{+}$ (resp., $\Sigma_{-}$) the set of simple roots of $\mathfrak{r}_{+}\left(\right.$resp., $\left.\mathfrak{r}_{-}\right)$with respect to the above Borel subalgebras. Let $\Sigma=\Sigma_{+} \cup \Sigma_{-}$and denote by $\mathcal{W}=\left(H_{\alpha}, X_{\alpha}, Y_{\alpha}\right)_{\alpha \in \Sigma_{+}}$a Weyl system of generators of $[\mathfrak{r}, \mathfrak{r}]$.

Definition 2.7 (Delorme, [2]). One calls $\left(A, A^{\prime}, \mathfrak{i}_{\mathfrak{a}}, \mathfrak{i}_{\mathfrak{a}^{\prime}}\right)$ generalized Belavin-Drinfeld data with respect to $B$ when the following five conditions are satisfied:

(1) $A$ is a bijection from a subset $\Gamma_{+}$of $\Sigma_{+}$on a subset $\Gamma_{-}$of $\Sigma_{-}$such that

$$
B\left(H_{A \alpha}, H_{A \beta}\right)=-B\left(H_{\alpha}, H_{\beta}\right), \alpha, \beta \in \Gamma_{+} .
$$

(2) $A^{\prime}$ is a bijection from a subset $\Gamma_{+}^{\prime}$ of $\Sigma_{+}$on a subset $\Gamma_{-}^{\prime}$ of $\Sigma_{-}$such that

$$
B\left(H_{A^{\prime} \alpha}, H_{A^{\prime} \beta}\right)=-B\left(H_{\alpha}, H_{\beta}\right), \alpha, \beta \in \Gamma_{+}^{\prime} .
$$

(3) If $C=A^{-1} A^{\prime}$ is the map defined on $\operatorname{dom}(\mathrm{C})=\left\{\alpha \in \Gamma_{+}^{\prime}: \mathrm{A}^{\prime} \alpha \in \Gamma_{-}\right\}$by $C \alpha=A^{-1} A^{\prime} \alpha$, then $C$ satisfies:

For all $\alpha \in \operatorname{dom}(\mathrm{C})$, there exists a positive integer $n$ such that $\alpha, \ldots, C^{n-1} \alpha \in \operatorname{dom}(\mathrm{C})$ and $C^{n} \alpha \notin$ $\operatorname{dom}(\mathrm{C})$.

(4) $\mathfrak{i}_{\mathfrak{a}}$ (resp., $\mathfrak{i}_{\mathfrak{a}^{\prime}}$ ) is a complex vector subspace of $\mathfrak{j}_{0}$, included and Lagrangian in the orthogonal $\mathfrak{a}$ (resp., $\mathfrak{a}^{\prime}$ ) to the subspace generated by $H_{\alpha}, \alpha \in \Gamma_{+} \cup \Gamma_{-}$(resp., $\Gamma_{+}^{\prime} \cup \Gamma_{-}^{\prime}$ ).

(5) If $\mathfrak{f}$ is the subspace of $\mathfrak{j}_{0}$ generated by the family $H_{\alpha}+H_{A \alpha}, \alpha \in \Gamma_{+}$, and $\mathfrak{f}^{\prime}$ is defined similarly, then

$$
\left(\mathfrak{f} \oplus \mathfrak{i}_{\mathfrak{a}}\right) \cap\left(\mathfrak{f}^{\prime} \oplus \mathfrak{i}_{\mathfrak{a}^{\prime}}\right)=0 .
$$

Let $R_{+}$be the set of roots of $\mathfrak{j}_{0}$ in $\mathfrak{r}$ which are linear combinations of elements of $\Gamma_{+}$. One defines similarly $R_{-}, R_{+}^{\prime}$ and $R_{-}^{\prime}$. The bijections $A$ and $A^{\prime}$ can then be extended by linearity to bijections from $R_{+}$to $R_{-}$(resp., $R_{+}^{\prime}$ to $R_{-}^{\prime}$ ). If $A$ satisfies condition (1), then there exists a unique isomorphism $\tau$ between the subalgebra $\mathfrak{m}_{+}$of $\mathfrak{r}$ spanned by $X_{\alpha}, H_{\alpha}$ and $Y_{\alpha}, \alpha \in \Gamma_{+}$, and the subalgebra $\mathfrak{m}_{-}$spanned by $X_{\alpha}, H_{\alpha}$ and $Y_{\alpha}, \alpha \in \Gamma_{-}$, such that $\tau\left(H_{\alpha}\right)=H_{A \alpha}, \tau\left(X_{\alpha}\right)=X_{A \alpha}, \tau\left(Y_{\alpha}\right)=Y_{A \alpha}$ for all $\alpha \in \Gamma_{+}$. If $A^{\prime}$ satisfies (2), then one defines similarly an isomorphism $\tau^{\prime}$ between $\mathfrak{m}^{\prime}+$ and $\mathfrak{m}^{\prime}{ }_{-}$.

Theorem 2.8 (Delorme, [2]). (i) Let $\mathcal{B D}=\left(A, A^{\prime}, \mathfrak{i}_{\mathfrak{a}}, \mathfrak{i}_{\mathfrak{a}^{\prime}}\right)$ be generalized Belavin-Drinfeld data, with respect to $B$. Let $\mathfrak{n}$ be the sum of the root spaces relative to roots $\alpha$ of $\mathfrak{j}_{0}$ in $\mathfrak{b}_{0}$, which are not in $R_{+} \cup R_{-}$. Let $\mathfrak{i}:=\mathfrak{k} \oplus \mathfrak{i}_{\mathfrak{a}} \oplus \mathfrak{n}$, where $\mathfrak{k}:=\left\{X+\tau(X): X \in \mathfrak{m}_{+}\right\}$.

Let $\mathfrak{n}^{\prime}$ be the sum of the root spaces relative to roots $\alpha$ of $\mathfrak{j}_{0}$ in $\mathfrak{b}_{0}^{\prime}$, which are not in $R_{+}^{\prime} \cup R_{-}^{\prime}$. Let $\mathfrak{i}^{\prime}:=\mathfrak{k}^{\prime} \oplus \mathfrak{i}_{\mathfrak{a}^{\prime}} \oplus \mathfrak{n}^{\prime}$, where $\mathfrak{k}^{\prime}:=\left\{X+\tau^{\prime}(X): X \in \mathfrak{m}^{\prime}{ }_{+}\right\}$.

Then $\left(B, \mathfrak{i}, \mathfrak{i}^{\prime}\right)$ is a Manin triple.

(ii) Every Manin triple is conjugate by an element of $\mathcal{R}$ to a unique Manin triple of this type.

Let us consider the particular case $\mathfrak{r}=L_{\alpha} \times \mathfrak{g}$. We set $\Sigma_{+}:=\left(\Gamma^{e x t} \backslash\{\alpha\}\right) \times\{0\}, \Sigma_{-}:=\{0\} \times \Gamma$ and $\Sigma:=\Sigma_{+} \cup \Sigma_{-}$.

Denote by $\left(X_{\gamma}, Y_{\gamma}, H_{\gamma}\right)_{\gamma \in \Gamma}$ a Weyl system of generators for $\mathfrak{g}$ with respect to the root system $\Gamma$. Denote $-\alpha_{\max }$ by $\alpha_{0}$. Let $H_{\alpha_{0}}$ be the coroot of $\alpha_{0}$. We choose $X_{\alpha_{0}} \in \mathfrak{g}_{\alpha_{0}}, Y_{\alpha_{0}} \in \mathfrak{g}_{-\alpha_{0}}$ such that $\left[X_{\alpha_{0}}, Y_{\alpha_{0}}\right]=H_{\alpha_{0}}$. 
A Weyl system of generators in $L_{\alpha} \times \mathfrak{g}$ (with respect to the root system $\Sigma$ ) is the following: $X_{(\beta, 0)}=$ $\left(X_{\beta}, 0\right), H_{(\beta, 0)}=\left(H_{\beta}, 0\right), Y_{(\beta, 0)}=\left(Y_{\beta}, 0\right)$, for any $\beta \in \Gamma^{e x t} \backslash\{\alpha\}$, and $X_{(0, \gamma)}=\left(0, X_{\gamma}\right), H_{(0, \gamma)}=$ $\left(0, H_{\gamma}\right), Y_{(0, \gamma)}=\left(0, Y_{\gamma}\right)$, for any $\gamma \in \Gamma$.

By applying the general result of Delorme, one can deduce the description of the Manin triples of the form $\left(Q^{\prime}, \Delta_{\alpha}, \mathfrak{l}\right)$.

First, let us denote by $i$ the embedding

$$
\Gamma \backslash\{\alpha\} \hookrightarrow \Gamma^{e x t} \backslash\{\alpha\},
$$

Recall that $\mathfrak{g}_{0}$ denotes the reductive part of $P_{\alpha}^{-}$and has the Dynkin diagram $\Gamma \backslash\{\alpha\}$. Then $i$ induces an inclusion

$$
\mathfrak{g}_{0} \hookrightarrow L_{\alpha} .
$$

We will also denote this embedding by $i$.

Corollary 2.9. Let $S:=\Gamma \backslash\{\alpha\}$ and $\zeta_{S}:=\{h \in \mathfrak{h}: \beta(h)=0, \forall \beta \in S\}$. For any Manin triple $\left(Q^{\prime}, \Delta_{\alpha}, \mathfrak{l}\right)$, there exists a unique generalized Belavin-Drinfeld data $\mathcal{B D}=\left(A, A^{\prime}, \mathfrak{i}_{\mathfrak{a}}, \mathfrak{i}_{\mathfrak{a}^{\prime}}\right)$ where $A$ : $i(S) \times\{0\} \longrightarrow\{0\} \times S, A(i(\gamma), 0)=(0, \gamma)$ and $\mathfrak{i}_{\mathfrak{a}}=\operatorname{diag}\left(\zeta_{\mathrm{S}}\right)$, such that $\left(Q^{\prime}, \Delta_{\alpha}, \mathfrak{l}\right)$ is conjugate to the Manin triple $\mathcal{T}_{\mathcal{B D}}=\left(Q^{\prime}, \mathfrak{i}, \mathfrak{i}^{\prime}\right)$. Moreover, up to a conjugation which preserves $\Delta_{\alpha}$, one has $\mathfrak{l}=\mathfrak{i}^{\prime}$.

Proof. Let us suppose that $\left(Q^{\prime}, \Delta_{\alpha}, \mathfrak{l}\right)$ is a Manin triple. Then there exists a unique generalized Belavin-Drinfeld data $\mathcal{B D}=\left(A, A^{\prime}, \mathfrak{i}_{\mathfrak{a}}, \mathfrak{i}_{\mathfrak{a}^{\prime}}\right)$ such that the corresponding $\mathcal{T}_{\mathcal{B D}}=\left(Q^{\prime}, \mathfrak{i}, \mathfrak{i}^{\prime}\right)$ is conjugate to $\left(Q^{\prime}, \Delta_{\alpha}, \mathfrak{l}\right)$. Since $\mathfrak{i}$ and $\Delta_{\alpha}$ are conjugate and $\Delta_{\alpha}$ is "under" the parabolic subalgebra $P_{\alpha_{0}}^{+} \times P_{\alpha}^{-}$, it follows that $\mathfrak{i}$ is also "under" this parabolic and thus $\mathfrak{a}=\zeta_{S} \times \zeta_{S}$. According to [2], p. 136, the map $A$ should be an isometry between $i(S) \times\{0\}$ and $\{0\} \times S$. Let us write $A(i(\gamma), 0)=(0, \tilde{A}(\gamma))$, where $\tilde{A}: S \rightarrow S$ is an isometry which will be determined below.

Let $\mathfrak{m}$ be the image of $\mathfrak{g}_{0}$ in $L_{\alpha}$ via the embedding $i$. Then $\mathfrak{m}$ is spanned by $X_{i(\beta)}, H_{i(\beta)}, Y_{i(\beta)}$ for all $\beta \in S$.

According to Theorem 2.8, the Lagrangian subspace $\mathfrak{i}$ contains $\mathfrak{k}:=\{(X, \tau(X)): X \in \mathfrak{m}\}$, where $\tau$ satisfies the following conditions: $\tau\left(X_{i(\beta)}\right)=X_{\tilde{A} \beta}, \tau\left(H_{i(\beta)}\right)=H_{\tilde{A} \beta}, \tau\left(Y_{i(\beta)}\right)=Y_{\tilde{A} \beta}$, for all $\beta \in S$.

We obtain $\tau i\left(X_{\beta}\right)=X_{\tilde{A} \beta}, \tau i\left(Y_{\beta}\right)=Y_{\tilde{A} \beta}, \tau i\left(H_{\beta}\right)=H_{\tilde{A} \beta}$, for all $\beta \in S$.

Since $\mathfrak{i}$ and $\Delta_{\alpha}$ are conjugate, we must have $\mathfrak{i}_{\mathfrak{a}}=\operatorname{diag}\left(\zeta_{\mathrm{S}}\right)$. Moreover $\tau i$ has to be an inner automorphism of $\mathfrak{g}_{0}$. It follows that the isometry $\tilde{A}: S \rightarrow S$ which corresponds to this inner automorphism must be the identity. Thus $\tilde{A}=$ id and this ends the proof.

We will consider triples of the form $\left(\Gamma_{1}^{\prime}, \Gamma_{2}^{\prime}, \tilde{A}^{\prime}\right)$, where $\Gamma_{1}^{\prime} \subseteq \Gamma^{e x t} \backslash\{\alpha\}, \Gamma_{2}^{\prime} \subseteq \Gamma$ and $\tilde{A}^{\prime}$ is an isometry between $\Gamma_{1}^{\prime}$ and $\Gamma_{2}^{\prime}$.

Definition 2.10. We say that a triple $\left(\Gamma_{1}^{\prime}, \Gamma_{2}^{\prime}, \tilde{A}^{\prime}\right)$ is of type $I$ if $\alpha \notin \Gamma_{2}^{\prime}$ and $\left(\Gamma_{1}^{\prime}, i\left(\Gamma_{2}^{\prime}\right), i \tilde{A}^{\prime}\right)$ is an admissible triple in the sense of [1]. The triple $\left(\Gamma_{1}^{\prime}, \Gamma_{2}^{\prime}, \tilde{A}^{\prime}\right)$ is of type II if $\alpha \in \Gamma_{2}^{\prime}$ and $\tilde{A}^{\prime}(\beta)=\alpha$, for some $\beta \in \Gamma_{1}^{\prime}$ and $\left(\Gamma_{1}^{\prime} \backslash\{\beta\}, i\left(\Gamma_{2}^{\prime} \backslash\{\alpha\}\right), i \tilde{A}^{\prime}\right)$ is an admissible triple in the sense of [1].

Using the definition of generalized Belavin-Drinfeld data, one can easily check the following:

Lemma 2.11. Let $A: i(S) \times\{0\} \longrightarrow\{0\} \times S, A(i(\gamma), 0)=(0, \gamma)$ and $\mathfrak{i}_{\mathfrak{a}}=\operatorname{diag}\left(\zeta_{\mathrm{S}}\right)$. A quadruple $\left(A, A^{\prime}, \mathfrak{i}_{\mathfrak{a}}, \mathfrak{i}_{\mathfrak{a}^{\prime}}\right)$ is generalized Belavin-Drinfeld data if and only if the pair $\left(A^{\prime}, \mathfrak{i}_{\mathfrak{a}^{\prime}}\right)$ satisfies the following conditions:

(1) $A^{\prime}: \Gamma_{1}^{\prime} \times\{0\} \longrightarrow\{0\} \times \Gamma_{2}^{\prime}$ is given by $A^{\prime}(\gamma, 0)=\left(0, \tilde{A}^{\prime}(\gamma)\right)$ and $\left(\Gamma_{1}^{\prime}, \Gamma_{2}^{\prime}, \tilde{A}^{\prime}\right)$ is of type $I$ or II from above.

(2) Let $\mathfrak{f}$ be the subspace of $\mathfrak{h} \times \mathfrak{h}$ spanned by pairs $\left(H_{i(\gamma)}, H_{\gamma}\right)$ for all $\gamma \in S$ and $\mathfrak{f}^{\prime}$ be the subspace of $\mathfrak{h} \times \mathfrak{h}$ spanned by pairs $\left(H_{\beta}, H_{\tilde{A}^{\prime}(\beta)}\right)$ for all $\beta \in \Gamma_{1}^{\prime}$. Let $\mathfrak{i}_{\mathfrak{a}^{\prime}}$ be Lagrangian subspace of $\mathfrak{a}^{\prime}:=\left\{\left(h_{1}, h_{2}\right) \in\right.$ $\left.\mathfrak{h} \times \mathfrak{h}: \beta\left(h_{1}\right)=0, \gamma\left(h_{2}\right)=0, \forall \beta \in \Gamma_{1}^{\prime}, \forall \gamma \in \Gamma_{2}^{\prime}\right\}$. Then

$$
\left(\mathfrak{f}^{\prime} \oplus \mathfrak{i}_{\mathfrak{a}^{\prime}}\right) \cap\left(\mathfrak{f} \oplus \mathfrak{i}_{\mathfrak{a}}\right)=0 .
$$

Remark 2.12. One can always find $\mathfrak{i}_{\mathfrak{a}^{\prime}}$ which is a Lagrangian subspace of $\mathfrak{a}^{\prime}$ and satisfies condition (2.6). This is a consequence of 2 Remark 2, p. 142. A proof of this elementary fact can also be found in [12, Lemma 5.2. 
Summing up the previous results we conclude the following:

Theorem 2.13. Let $\alpha$ be a simple root. Suppose that $\mathfrak{l}$ is a Lagrangian subalgebra of $L_{\alpha} \times \mathfrak{g}$ transversal to $\Delta_{\alpha}$. Then, up to a conjugation which preserves $\Delta_{\alpha}$, one has $\mathfrak{l}=\mathfrak{i}^{\prime}$, where $\mathfrak{i}^{\prime}$ is constructed from a pair formed by a triple $\left(\Gamma_{1}^{\prime}, \Gamma_{2}^{\prime}, \tilde{A}^{\prime}\right)$ of type I or II and a Lagrangian subspace $\mathfrak{i}_{\mathfrak{a}^{\prime}}$ of $\mathfrak{a}^{\prime}$ such that (2.6) is satisfied.

Remark 2.14. The above algorithm covers also the case when the simple root $\alpha$ has coefficient $k=1$ in the decomposition of $\alpha_{\max }$, which was considered in [7. In this form we do not need to use the Cartan involution which appeared there.

Remark 2.15. In [7] we constructed two examples of quasi-trigonometric solutions for $\mathfrak{g}=\mathfrak{s l}(n+1)$ related to the Cremmer-Gervais $r$-matrix. Let us apply the present algorithm for the simple root $\alpha_{n}$. Let $\Gamma=\left\{\alpha_{1}, \ldots, \alpha_{n}\right\}$ and $\alpha_{0}=-\alpha_{\max }$.

We consider the triple $\left(\Gamma_{1}^{\prime}, \Gamma_{2}^{\prime}, \tilde{A}^{\prime}\right)$, where $\Gamma_{1}^{\prime}=\left\{\alpha_{0}, \alpha_{1}, \ldots \alpha_{n-2}\right\}, \Gamma_{2}^{\prime}=\left\{\alpha_{1}, \ldots \alpha_{n-1}\right\}$ and $\tilde{A}^{\prime}\left(\alpha_{j}\right)=$ $\alpha_{j+1}$, for $j=0, \ldots, n-2$. This is a triple of type I, cf. Definition 2.10.

Another example is given by the following triple: $\Gamma_{1}^{\prime}=\left\{\alpha_{0}, \alpha_{1}, \ldots \alpha_{n-1}\right\}, \Gamma_{2}^{\prime}=\left\{\alpha_{1}, \ldots \alpha_{n}\right\}$ and $\tilde{A}^{\prime}\left(\alpha_{j}\right)=\alpha_{j+1}$, for $j=1, \ldots, n-1$. This is a triple of type II, cf. Definition 2.10,

The above triples together with the corresponding subspaces $\mathfrak{i}_{\mathfrak{a}^{\prime}}$ (which we do not compute here) induce two Lagrangian subalgebras of Cremmer-Gervais type.

Theorem 2.13 can also be applied to roots which have coefficient greater than one. As an example, let us classify solutions in $\mathfrak{g}=o(5)$.

Corollary 2.16. Let $\alpha_{1}, \alpha_{2}$ be the simple roots in o(5) and $\alpha_{0}=-2 \alpha_{1}-\alpha_{2}$. Up to gauge equivalence, there exist two quasi-trigonometric solutions with non-trivial polynomial part.

Proof. The root $\alpha_{2}$ has coefficient $k=1$ in the decomposition of the maximal root. The only possible choice for a triple $\left(\Gamma_{1}^{\prime}, \Gamma_{2}^{\prime}, \tilde{A}^{\prime}\right)$ with $\Gamma_{1}^{\prime} \subseteq\left\{\alpha_{0}, \alpha_{1}\right\}, \Gamma_{2}^{\prime} \subseteq\left\{\alpha_{1}, \alpha_{2}\right\}$ to be of type I or II is $\Gamma_{1}^{\prime}=\left\{\alpha_{0},\right\}$, $\Gamma_{2}^{\prime}=\left\{\alpha_{2},\right\}, \tilde{A}^{\prime}\left(\alpha_{0}\right)=\alpha_{2}$. One can check that $\mathfrak{i}_{\mathfrak{a}^{\prime}}$ is a 1-dimensional space spanned by the following pair $(\operatorname{diag}(-2,1,0,-1,2), \operatorname{diag}(0, \sqrt{5}, 0,-\sqrt{5}, 0))$. The Lagrangian subalgebra $\mathfrak{i}_{2}^{\prime}$ constructed from this triple is transversal to $\Delta_{\alpha_{2}}$ in $\mathfrak{g} \times \mathfrak{g}$.

The root $\alpha_{1}$ has coefficient $k=2$ in the decomposition of the maximal root. The only possible choice for a triple $\left(\Gamma_{1}^{\prime}, \Gamma_{2}^{\prime}, \tilde{A}^{\prime}\right)$ with $\Gamma_{1}^{\prime} \subseteq\left\{\alpha_{0}, \alpha_{2}\right\}, \Gamma_{2}^{\prime} \subseteq\left\{\alpha_{1}, \alpha_{2}\right\}$ is again $\Gamma_{1}^{\prime}=\left\{\alpha_{0},\right\}, \Gamma_{2}^{\prime}=\left\{\alpha_{2},\right\}$, $\tilde{A}^{\prime}\left(\alpha_{0}\right)=\alpha_{2}$ and $\mathfrak{i}_{\mathfrak{a}^{\prime}}$ is as in the previous case. The Lagrangian subalgebra $\mathfrak{i}_{1}^{\prime}$ constructed from this triple is transversal to $\Delta_{\alpha_{1}}$ in $L_{\alpha_{1}} \times \mathfrak{g}$.

\section{QUANTIZATION OF QUASI-TRIGONOMETRIC SOlUTIONS}

Let us consider the Lie bialgebra structure on $\mathfrak{g}[u]$ given by the simplest quasi-trigonometric solution:

$$
\delta(p(u))=[r(u, v), p(u) \otimes 1+1 \otimes p(v)],
$$

with $r(u, v)=v \Omega /(u-v)+\Sigma_{\alpha} e_{\alpha} \otimes f_{\alpha}+\frac{1}{2} \Omega_{0}$, where $e_{\alpha}, f_{\alpha}$ are root vectors of $\mathfrak{g}$ and $\Omega_{0}$ is the Cartan part of $\Omega$. Here $\delta$ and $r$ are exactly the same as $\delta_{3}$ and $r_{3}$ from the Introduction.

Quasi-trigonometric solutions correspond to Lie bialgebra structures on $\mathfrak{g}[u]$ which are obtained by twisting $\delta$. The quantization of the Lie bialgebra $(\mathfrak{g}[u], \delta)$ is the quantum algebra $U_{q}(\mathfrak{g}[u])$ introduced by V. Tolstoy in [14. We will recall its construction with a slight modification.

Let $\mathfrak{g}$ be a finite-dimensional complex simple Lie algebra of rank $l$ with a standard Cartan matrix $A=\left(a_{i j}\right)_{i, j=1}^{l}$, with a system of simple roots $\Gamma=\left\{\alpha_{1}, \ldots, \alpha_{l}\right\}$, and with a Chevalley basis $h_{\alpha_{i}}, e_{ \pm \alpha_{i}}$ $(i=1,2, \ldots, l)$. Let $\theta$ be the maximal (positive) root of $\mathfrak{g}$. The corresponding non-twisted affine algebra with zero central charge $\hat{\mathfrak{g}}$ is generated by $\mathfrak{g}$ and the additional affine elements $e_{\delta-\theta}:=u e_{-\theta}$, $e_{-\delta+\theta}:=u^{-1} e_{\theta}$.

The Lie algebra $\mathfrak{g}[u]$ is generated by $\mathfrak{g}$, the positive root vector $e_{\delta-\theta}$ and the Cartan element $h_{\delta-\theta}=\left[e_{\delta-\theta}, e_{-\delta+\theta}\right]$. The standard defining relations of the universal enveloping algebra $U(\mathfrak{g}[u])$ are 
given by the formulas:

$$
\begin{aligned}
{\left[h_{\alpha_{i}}, h_{\alpha_{j}}\right] } & =0, \\
{\left[h_{\alpha_{i}}, e_{ \pm \alpha_{j}}\right] } & = \pm\left(\alpha_{i}, \alpha_{j}\right) e_{ \pm \alpha_{j}}, \\
{\left[e_{\alpha_{i}}, e_{-\alpha_{j}}\right] } & =\delta_{i j} h_{\alpha_{i}}, \\
\left(\operatorname{ad} e_{ \pm \alpha_{i}}\right)^{n_{i j}} e_{ \pm \alpha_{j}} & =0 \quad \text { for } i \neq j, \quad n_{i j}:=1-a_{i j}, \\
{\left[h_{\alpha_{i}}, e_{\delta-\theta}\right] } & =-\left(\alpha_{i}, \theta\right) e_{\delta-\theta}^{\prime}, \\
{\left[e_{-\alpha_{i}}, e_{\delta-\theta}\right] } & =0, \\
\left(\operatorname{ad} e_{\alpha_{i}}\right)^{n_{i 0}} e_{\delta-\theta} & =0 \quad \text { for } n_{i 0}=1+2\left(\alpha_{i}, \theta\right) /\left(\alpha_{i}, \alpha_{i}\right), \\
{\left[\left[e_{\alpha_{i}}, e_{\delta-\theta}\right], e_{\delta-\theta}\right] } & =0 \quad \text { for } \mathfrak{g} \neq \mathfrak{s l}_{2} \quad \text { and }\left(\alpha_{i}, \theta\right) \neq 0, \\
{\left[\left[\left[e_{\alpha}, e_{\delta-\alpha}\right], e_{\delta-\alpha}\right], e_{\delta-\alpha}\right] } & =0 \quad \text { for } \mathfrak{g}=\mathfrak{s l}_{2} \quad(\theta=\alpha) .
\end{aligned}
$$

The quantum algebra $U_{q}(\mathfrak{g}[u])$ is a $q$-deformation of $U(\mathfrak{g}[u])$. The Chevalley generators for $U_{q}(\mathfrak{g}[u])$ are $k_{\alpha_{i}}^{ \pm 1}:=q^{ \pm h_{\alpha_{i}}}, e_{ \pm \alpha_{i}}(i=1,2, \ldots, l), e_{\delta-\theta}$ and $k_{\delta-\theta}:=q^{h_{\delta-\theta}}$. Then the defining relations of $U_{q}(\mathfrak{g}[u])$ are the following:

$$
\begin{aligned}
k_{\alpha_{i}}^{ \pm 1} k_{\alpha_{j}}^{ \pm 1} & =k_{\alpha_{j}}^{ \pm 1} k_{\alpha_{i}}^{ \pm 1}, \\
k_{\alpha_{i}} k_{\alpha_{i}}^{-1} & =k_{\alpha_{i}}^{-1} k_{\alpha_{i}}=1, \\
k_{\alpha_{i}} e_{ \pm \alpha_{j}} k_{\alpha_{i}}^{-1} & =q^{ \pm\left(\alpha_{i}, \alpha_{j}\right)} e_{ \pm \alpha_{j}}, \\
{\left[e_{\alpha_{i}}, e_{-\alpha_{i}}\right] } & =\frac{k_{\alpha_{i}}-k_{\alpha_{i}}^{-1}}{q-q^{-1}}, \\
\left(\operatorname{ad}_{q} e_{ \pm \alpha_{i}}\right)^{n_{i j}} e_{ \pm \alpha_{j}} & =0 \text { for } i \neq j, \quad n_{i j}:=1-a_{i j}, \\
k_{\alpha_{i}} e_{\delta-\theta} k_{\alpha_{i}}^{-1} & =q^{-\left(\alpha_{i}, \theta\right)} e_{\delta-\theta}, \\
{\left[e_{-\alpha_{i}}, e_{\delta-\theta}\right] } & =0, \\
\left(\operatorname{ad}_{q} e_{\alpha_{i}}\right)^{n_{i 0}} e_{\delta-\theta} & =0 \text { for } n_{i 0}=1+2\left(\alpha_{i}, \theta\right) /\left(\alpha_{i}, \alpha_{i}\right), \\
{\left[\left[e_{\alpha_{i}}, e_{\delta-\theta}\right]_{q}, e_{\delta-\theta}\right]_{q} } & =0 \text { for } \mathfrak{g} \neq \mathfrak{s l}_{2} \text { and }\left(\alpha_{i}, \theta\right) \neq 0, \\
{\left.\left[\left[e_{\alpha}, e_{\delta-\alpha}\right]_{q}, e_{\delta-\alpha}\right]_{q}, e_{\delta-\alpha}\right]_{q} } & =0 \text { for } \mathfrak{g}=\mathfrak{s l}_{2},
\end{aligned}
$$

where $\left(\operatorname{ad}_{q} e_{\beta}\right) e_{\gamma}$ is the q-commutator:

$$
\left(\operatorname{ad}_{q} e_{\beta}\right) e_{\gamma}:=\left[e_{\beta}, e_{\gamma}\right]_{q}:=e_{\beta} e_{\gamma}-q^{(\beta, \gamma)} e_{\gamma} e_{\beta}
$$

The comultiplication $\Delta_{q}$, the antipode $S_{q}$, and the co-unit $\varepsilon_{q}$ of $U_{q}(\mathfrak{g}[u])$ are given by

$$
\begin{aligned}
\Delta_{q}\left(k_{\alpha_{i}}^{ \pm 1}\right) & =k_{\alpha_{i}}^{ \pm 1} \otimes k_{\alpha_{i}}^{ \pm 1} \\
\Delta_{q}\left(e_{-\alpha_{i}}\right) & =e_{-\alpha_{i}} \otimes k_{\alpha_{i}}+1 \otimes e_{-\alpha_{i}}, \\
\Delta_{q}\left(e_{\alpha_{i}}\right) & =e_{\alpha_{i}} \otimes 1+k_{\alpha_{i}}^{-1} \otimes e_{\alpha_{i}}, \\
\Delta_{q}\left(e_{\delta-\theta}\right) & =e_{\delta-\theta} \otimes 1+k_{\delta-\theta}^{-1} \otimes e_{\delta-\theta},
\end{aligned}
$$




$$
\begin{aligned}
S_{q}\left(k_{\alpha_{i}}^{ \pm 1}\right) & =k_{\alpha_{i}}^{\mp 1}, \\
S_{q}\left(e_{-\alpha_{i}}\right) & =-e_{-\alpha_{i}} k_{\alpha_{i}}^{-1}, \\
S_{q}\left(e_{\alpha_{i}}\right) & =-k_{\alpha_{i}} e_{\alpha_{i}}, \\
S_{q}\left(e_{\delta-\theta}\right) & =-k_{\delta-\theta} e_{\delta-\theta}, \\
\varepsilon_{q}\left(e_{ \pm \alpha_{i}}\right)=\varepsilon_{q}\left(e_{\delta-\theta}\right) & =0, \quad \varepsilon_{q}\left(k_{\alpha_{i}}^{ \pm 1}\right)=1 .
\end{aligned}
$$

We see that

$$
k_{\delta-\theta}=k_{\alpha_{1}}^{-n_{1}} k_{\alpha_{2}}^{-n_{2}} \cdots k_{\alpha_{l}}^{-n_{l}}
$$

if $\theta=n_{1} \alpha_{1}+n_{2} \alpha_{2}+\cdots+n_{l} \alpha_{l}$.

Let us consider now $U_{\hbar}(\mathfrak{g}[u])$, which is an algebra over $\left.\mathbb{C}[\hbar]\right]$ defined by the previous relations in which we set $q=\exp (\hbar)$.

Theorem 3.1. The classical limit of $U_{\hbar}(\mathfrak{g}[u])$ is $(\mathfrak{g}[u], \delta)$.

Proof. Since the image of $e_{\delta-\theta}$ in $U_{\hbar}(\mathfrak{g}[u]) / \hbar U_{\hbar}(\mathfrak{g}[u])$ is $u e_{\theta}$, we can identify the classical limit of $U_{\hbar}(\mathfrak{g}[u])$ with $U(\mathfrak{g}[u])$.

It remains to prove that for any $a \in \mathfrak{g}[u]$ and its preimage $\tilde{a} \in U_{\hbar}(\mathfrak{g}[u])$, we have

$$
\hbar^{-1}\left(\Delta(\tilde{a})-\Delta^{o p}(\tilde{a})\right) \bmod \hbar=\delta(a) .
$$

It is clear that $U_{\hbar}(\mathfrak{g}[u])$ is a Hopf subalgebra of $U_{\hbar}(\hat{\mathfrak{g}})$ with zero central charge. It follows from [3] that $\Delta^{o p}(\tilde{a})=R \Delta(\tilde{a}) R^{-1}$, where $R=1 \otimes 1+\hbar r+\ldots$ is the universal $R$-matrix for $U_{\hbar}(\hat{\mathfrak{g}})$ (see also [8] and $[5])$.

Writing $\tilde{a}=a+\hbar s$, we see that

$$
\hbar^{-1}\left(\Delta(\tilde{a})-R \Delta(a) R^{-1}\right) \bmod \hbar=[r, a \otimes 1+1 \otimes a]=\delta(a) .
$$

This ends the proof of the theorem.

The Lie bialgebra structures induced by quasi-trigonometric solutions are obtained by twisting $\delta$ via a classical twist. According to Theorem 2.13 any such twist is given by a triple $\left(\Gamma_{1}^{\prime}, \Gamma_{2}^{\prime}, \tilde{A}^{\prime}\right)$ of type I or II together with a Lagrangian subspace $\mathfrak{i}_{\mathfrak{a}^{\prime}}$ of $\mathfrak{a}^{\prime}$ such that (2.6) is satisfied.

On the other hand, Theorem 1.1] states that classical twists can be extended to quantum twists.

We conclude that any data of the form $\left(\Gamma_{1}^{\prime}, \Gamma_{2}^{\prime}, \tilde{A}^{\prime}, \mathfrak{i}_{\mathfrak{a}^{\prime}}\right)$ provides a twisted comultiplication and antipode in the quantum algebra $U_{\hbar}(\mathfrak{g}[u])$. In case $\mathfrak{g}=\mathfrak{s l}(n)$, some exact formulas were obtained in [7.

Acknowledgement. The authors are thankful to V. Tolstoy for valuable discussions.

\section{REFERENCES}

[1] A. Belavin, V. Drinfeld, Triangle equations and simple Lie algebras. Math. Phys. Reviews, Vol. 4 (1984), Harwood Academic, 93-165.

[2] P. Delorme, Classification des triples de Manin pour les algèbres de Lie réductives complexes. J. Algebra, 246 (2001), 97-174.

[3] V. Drinfeld, Quantum groups. Proceedings ICM (Berkeley 1986) 1 (1987), Amer. Math. Soc., 798-820.

[4] G. Halbout, Formality theorem for Lie bialgebras and quantization of twists and coboundary r-matrices. Adv. Math. 207 (2006), 617-633.

[5] M. Jimbo, Quantum R-matrix for the generalized Toda system, Comm. Math. Phys. 102, no.1 (1986), 537-547.

[6] S. Khoroshkin, I. Pop, A. Stolin, V. Tolstoy, On some Lie bialgebra structures on polynomial algebras and their quantization. Preprint no. 21, 2003/2004, Mittag-Leffler Institute, Sweden.

[7] S. Khoroshkin, I. Pop, M. Samsonov, A. Stolin, V. Tolstoy, On some Lie bialgebra structures on polynomial algebras and their quantization. ArXiv math. QA/0706.1651v1. To appear in Comm. Math. Phys.

[8] S. Khoroshkin, V. Tolstoy, Universal R-matrix for quantized (super) algebras. Comm. Math. Phys. 141 (1991), 599-617. 
[9] F. Montaner, E. Zelmanov, Bialgebra structures on current Lie algebras. Preprint, University of Wisconsin, Madison, 1993.

[10] A. Stolin, On rational solutions of Yang-Baxter equations. Maximal orders in loop algebra. Comm. Math. Phys. 141 (1991), 533-548.

[11] A. Stolin, A geometrical approach to rational solutions of the classical Yang-Baxter equation. Part I. Symposia Gaussiana, Conf.A, Walter de Gruyter, Berlin, New York, 1995, 347-357.

[12] A. Stolin, Some remarks on Lie bialgebra structures for simple complex Lie algebras. Comm. Alg. 27 (9) (1999), 4289-4302.

[13] A. Stolin, J. Yermolova-Magnusson, The 4th structure. Czech. J. Phys, Vol. 56, No. 10/11 (2006), $1293-1927$.

[14] V. Tolstoy, From quantum affine Kac-Moody algebras to Drinfeldians and Yangians. in Kac-Moody Lie algebras and related topics, Contemp. Math. 343, Amer. Math. Soc. 2004, 349-370.

Department of Mathematical Sciences, University of Gothenburg, Sweden

E-mail address: iulia@math.chalmers.se, astolin@math.chalmers.se 